\title{
Statistical Approach to ML Decoding of Linear Block Codes on Symmetric Channels
}

\author{
Haris Vikalo and Babak Hassibi ${ }^{1}$ \\ Department of Electrical Engineering \\ California Institute of Technology \\ $\{$ hvikalo, hassibi\}@systems. caltech.edu
}

\begin{abstract}
Maximum-likelihood (ML) decoding of linear block codes on a symmetric channel is studied. Exact ML decoding is known to be computationally difficult. We propose an algorithm that finds the exact solution to the ML decoding problem by performing a depth-first search on a tree. The tree is designed from the code generator matrix and pruned based on the statistics of the channel noise. The complexity of the algorithm is a random variable. We characterize the complexity by means of its first moment, which for binary symmetric channels we find in closed-form. The obtained results indicate that the expected complexity of the algorithm is low over a wide range of system parameters.
\end{abstract}

\section{SUMmary}

We consider transmission over the q-ary symmetric channel. The channel encoder maps the $m \times 1$ information data vector $\mathbf{b}$ into the $n \times 1$ codeword $\mathbf{c}$. The encoder employs linear mapping defined via an $n \times m$ code generator matrix $\mathbf{G}$, i.e., $\mathbf{c}=\mathbf{G} \cdot \mathbf{b}$. The receiver observes a corrupted version of the transmitted codeword, $\mathbf{r}$, from which it attempts to recover the information vector $\mathbf{b}$. When the noise is additive, i.e., $\mathbf{r}=\mathbf{c}+\mathbf{v}$, the $M L$ decoding is equivalent to the nearest codeword problem,

$$
\min _{\mathbf{b}}|\mathbf{r}-\mathbf{G} \cdot \mathbf{b}|,
$$

where $|\cdot|$ denotes Hamming distance. The nearest codeword problem (1) is known to be NP-hard [1].

We propose an algorithm that solves (1) by finding valid codewords within certain Hamming distance $d$ from the observed vector $\mathbf{r}$, i.e., by finding $\mathbf{b}$ such that $|\mathbf{r}-\mathbf{G} \cdot \mathbf{b}| \leq d$. We can choose $d$ according to the statistics of $|\mathbf{v}|$. For brevity, we focus on a binary symmetric channel (BSC). Note that $|\mathbf{r}-\mathbf{G} \cdot \mathbf{b}|=|\mathbf{v}|=\sum_{i=1}^{n} v_{i}$. Since each $v_{i}$ is $\operatorname{Bernoulli}(p),|\mathbf{v}|$ has a binomial distribution and we choose $d$ so that

$$
\sum_{k=0}^{d}\left(\begin{array}{c}
n \\
l
\end{array}\right) p^{k}(1-p)^{n-k}=1-I_{p}(d+1, n-d)=1-\epsilon,
$$

where we set $1-\epsilon$ to be close to 1 (so that solution is found with high probability), where $I_{x}(a, b)=\frac{B(x ; a, b)}{B(a, b)}$ for $a \leq b$ and $I_{x}(a, b)=1$ otherwise, and where $B(a, b)$ is the beta function, and $B(x ; a, b)$ is the incomplete beta function.

Pre-process the code generator matrix $\mathbf{G}$ to an approximately upper-triangular form with a diagonal profile as defined by the set of ratios $\mathcal{D}=\left\{g_{1}^{(v)} / g_{1}^{(h)}, \ldots, g_{D}^{(v)} / g_{D}^{(h)}\right\}$, where

${ }^{1}$ This work was supported in part by the National Science Foundation under grant no. CCR-0133818, by the Office of Naval Research under grant no. N00014-02-1-0578, and by Caltech's Lee Center for Advanced Networking.

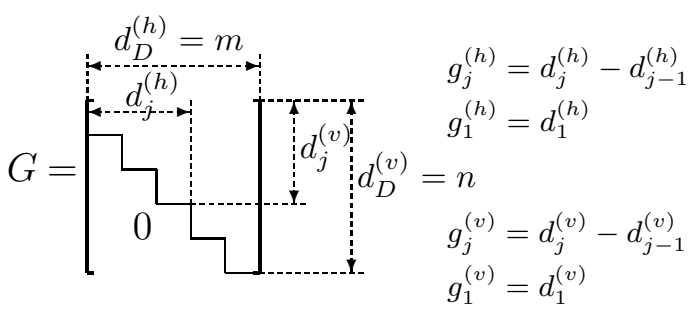

Now $|\mathbf{r}-\mathbf{G} \cdot \mathbf{b}| \leq d$ can be written as

$$
\sum_{j=1}^{D}\left|\mathbf{r}_{j}-G_{j j} \cdot \mathbf{b}_{j}+\sum_{k=j+1}^{D} G_{j k} \cdot \mathbf{b}_{k}\right| \leq d,
$$

where $G_{j k}=G\left(d_{j-1}^{(v)}+1: d_{j}^{(v)} ; d_{k-1}^{(h)}+1: d_{k}^{(h)}\right), \mathbf{b}_{j}=$ $\left[\begin{array}{lll}b_{d_{j-1}^{(h)}+1} & \cdots & b_{d_{j}^{(h)}}\end{array}\right]^{T}$, and where $\mathbf{r}_{j}=\left[\begin{array}{llll}r_{d_{j-1}^{(v)}+1} & \ldots & \mathbf{r}_{d_{j}^{(v)}}\end{array}\right]^{T}$, $j=1,2, \ldots, D, j \leq k \leq D$. We solve (3) with a constrained depth-first tree search similar in spirit to the one in [2]. If no points within distance $d$ is found, $d$ is increased (say, by decreasing $\epsilon$ in (2)) and the algorithm is run anew.

The complexity of the algorithm depends on $G$ and $\mathbf{v}$ and is thus a random variable. Let $f_{p}(k)$ denote the number of computation per tree node on level $k$. For $G$ with random $\operatorname{Bernoulli}\left(\frac{1}{2}\right)$ entries, expected complexity is given by

$$
\begin{aligned}
C(G, p)= & \sum_{k=1}^{D} f_{p}(k)\left[1-I_{p}\left(d+1, d_{k}^{(v)}-d\right)\right. \\
& \left.+\left(2^{d_{k}^{(h)}}-1\right)\left(1-I_{\frac{1}{2}}\left(d+1, d_{k}^{(v)}-d\right)\right)\right]
\end{aligned}
$$

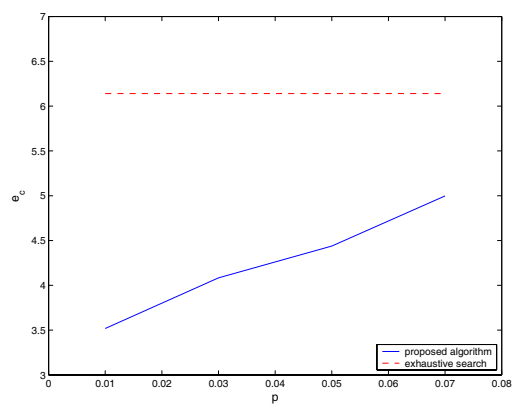

Figure 1: Expected complexity exponent of decoding $(R=$ $1 / 2, m=15, n=30$ ) random binary code.

Figure 1 illustrates expected complexity exponent of the algorithm, defined as $c_{e}=\log _{m}$ (average flopcount), and compares it with exhaustive search. For small $p$ (say, $p<0.01$ ), the expected complexity of the algorithm is roughly cubic.

\section{REFERENCES}

[1] E. R. Berlekamp, R. J. McEliece, and H. C. A. van Tilborg, "On the inherent intractability of certain coding problems," IEEE Transactions on Information Theory, 24(3):384-386, May 1978.

[2] U. Fincke and M. Pohst, "Improved methods for calculating vectors of short length in a lattice, including a complexity analysis," Mathematics of Comput., vol. 44, pp. 463-471, April 1985. 